УДК 621.314.21-027.3.04

\title{
ЭКСПЕРИМЕНТАЛЬНОЕ ИССЛЕДОВАНИЕ КОНТРОЛЯ СОСТОЯНИЯ ОБМОТОК ВЫСОКОВОЛЬТНЫХ ТРАНСФОРМАТОРОВ НА ОСНОВЕ КОММУТАЦИОННЫХ ИМПУЛЬСОВ
}

\author{
Лавринович Валерий Александрович1, \\ lavrhome@mail.ru
}

\author{
Лавринович Алексей Валериевич2, \\ lavrinovich86@yandex.ru
}

\author{
Мытников Алексей Владимирович³, \\ mytnikov66@mail.ru
}
1 Российский Федеральный Ядерный Центр - Всероссийский Научно-Исследовательский Институт Технической Физики - Всероссийский Электротехнический Институт, Россия, 111250, г. Москва, ул. Красноказарменная, 12.
2 Томский научно-исследовательский и проектный институт нефрти и газа, Россия, 634027, г. Томск, пр. Мира, 72.
3 Национальный исследовательский Томский политехнический университет, Россия, 634050, г. Томск, пр. Ленина, 30.

\begin{abstract}
Актуальность исследования. На всех этапах технологической цепи добычи, транспортировки и последующего использования геологических ресурсов применяется широкий спектр высоковольтного оборудования, существенную часть которого составляют высоковольтные трансформаторы различных типов, назначений и классов напряжения. Поэтому общая энергетическая эфффективность и надежность производства геологических ресурсов существенно зависит от состояния высоковольтного трансформаторного оборудования. Дефектное состояние одной трансфоорматорной единицы часто является причиной внезапной аварийной остановки всей технологической цепи комплексного производственного процесса. Результатом подобного инцидента является пожар, сопровождаемый взрывом высоковольтного ввода, разлив масла, загрязнение окружающей среды и материальный ущерб, исчисляемьй многомиллионными суммами. К возникновению дефектного и, как следствие, аварийного состояния приводит ряд электрофизических и эксплуатационных факторов, воздействующих на изоляцию и активную часть транссрорматоров. В свою очередь наиболее проблемным элементом активной части трансформатора оказываются обмотки как высокого, так и низкого напряжения, в которых появляются механические смещения как результат воздействия пондеромоторных сил токов короткого замыкания. Подобные дефректы могут развиваться длительное время, и трансформатор может находиться в работе в номинальном режиме. На некотором этапе развития дефект становится критическим, повреждения изоляции обмотки при очередном коротком замыкании ведут к возгоранию $\mathrm{c}$ последующей аварией. Для предупреждения подобных ситуаций необходима эфффективная технология контроля состояния обмоток. Стандартные методы являются малоэфффективными для выявления дефектов обмотки на ранних стадиях развития. Среди новых и рекомендованных международными экспертными институтами МЭК и СИГРЭ являются модисрикации импульсного метода, прежде всего метод частотного анализа. Однако, не смотря на признание как наиболее достоверного и перспективного, указанный метод далеко не всегда позволяет обнаружить дефектты обмоток, особенно на ранних этапах развития. Кроме того, обязательным условием применения технологии метода частотного анализа является полное снятие напряжения, расшиновка трансформатора и использование специальной аппаратуры контроля. При этом для получения достоверных результатов диагностики необходимо наличие специальных эталонных сигналов - нормограмм, которые отсутствуют практически для всего парка трансформаторов, используемых в технологических процессах добычи и переработки геологических ресурсов. Эти фракторы снижают общую эфффективность технологии метода частотного анализа. Поэтому в настоящее время вопрос по исследованию и разработке технологии контроля состояния обмоток под рабочим напряжением является в полной мере актуальным.

Цель: экспериментальное исследование принципиальной возможности контроля состояния обмоток трансформатора на основе процессов коммутации в высоковольтной сети.

Методы: метод моделирования на реальном физическом объекте. В трансформаторе НТМИ-6 на одной фазе созданы дефекты механического смещения обмоток. Две другие фазы имеют исправное состояние. При помощи специальной схемы трансформатор включался в сеть. С помощью электронного осциллографра Tektronix muna TDS-2012 осуществлялась регистрация напряжения на первичной обмотке и сигнала отклика с одноименной фразы вторичной обмотки. Измерения повторялись для двух других фраз по такой же схеме.

Результаты. На реальном трансформаторе типа НТМИ-6 экспериментально исследована и установлена принципиальная возможность осуществления контроля механического состояния его обмоток при использовании отклика напряжения на вторичной обмотке трансфоорматора при включении трансфрорматора в сеть. Таким образом, показано, что при включении транссрорматора в сеть подключаемое напряжение может служить диагностическим импульсом, пригодным для зондирова-
\end{abstract} ния механического состояния обмоток трансфформатора по методу низковольтных импульсов.

\section{Ключевые слова:}

Контроль состояния, импульсный метод, дефект обмотки, эфффективность диагностики. 


\section{Введение}

Большая часть энергетического оборудования, применяемого в горнорудной и нефтегазовой промышленности для добычи, транспортировки и переработки природных ресурсов, выработала свой номинальный срок службы, но продолжает находиться в состоянии интенсивной эксплуатации. Такая ситуация создается совокупностью экономических и технических факторов. Недостаток финансовых средств существенно сдерживает, а в ряде случаев не позволяет осуществлять замену высоковольтного оборудования с дефектным состоянием, срок эксплуатации которого завершен. Технический фактор заключается в отсутствии пригодных по всем критериям современных средств и технологий контроля состояния электрооборудования. Эффективность существующих методов диагностики электрооборудования во многих случаях не отвечает требованиям сегодняшнего дня.

Из-за старения целого поколения силовых трансформаторов сегодняшняя электроэнергетика столкнулась с серьезными проблемами, так как отказы трансформаторов, их ремонт и соответствующие затраты приводят к значительным экономическим потерям. Трансформаторы стали наиболее проблемными элементами электрических сетей. Потребность в надежных методах контроля и диагностики привела ведущих экспертов в этой области к необходимости разработки новых технологий, которые позволяют значительно увеличить надежность и оптимизировать работу каждого из элементов сети. Выход из строя такого объекта энергосистемы, как силовой трансформатор, часто приводит к аварии с тяжелыми последствиями. Одной из основных причин подобной аварийной ситуации являются изменения механического состояния обмоток трансформатора, такие как выпучивание витков, сдвиг витков в аксиальном и радиальном направлениях, распрессовка обмотки и т. п.

Существующие технологии диагностики далеко не всегда позволяют выявлять дефекты активных частей, и, как результат, число аварий растет. Так, по данным, представленным на IV Всероссийской научнотехнической конференции ведущими специалистами по диагностике силового электрооборудования, существующие технологи контроля трансформаторов выявляют не более $10 \%$ дефектов $[1,2]$. При этом статистический анализ показывает, что достаточно часто имеет место необоснованная отбраковка вполне работоспособного оборудования $[3,4]$. К наиболее точным и достоверным технологиям контроля состояния обмоток трансформаторов относятся метод низковольтных импульсов и метод частотных характеристик.

Метод низковольтных импульсов (НВИ) был исследован и доведен до практического применения в Институте электротехники, Варшава, Польша в 1966 г. Идея метода НВИ состоит в использовании импульса относительно низкого (200-400 В) напряжения, поступающего на обмотку обследуемого трансформатоpa, и снятия импульсного сигнала с соседней обмотки. Подаваемый на обмотку импульс получил название «зондирующий импульс». Импульсный сигнал, снимаемый с одной из ряда других обмоток, вызывается появлением зондирующего импульса и называется «сигнал отклика», или «отклик». Сигнал отклика представляет собой результат переходного процесса, вызванного зондирующим импульсом. В результате формирования дефектного состояния обмотки характеристики переходного процесса изменяются и, соответственно, изменяется форма сигнала откликам. Сравнение формы откликов, снятых по идентичной схеме при одинаковых параметрах зондирующего импульса, позволяет сделать вывод о наличии или отсутствии дефекта в обмотке. Определение наличия дефектного состояния на основе сравнения откликов лежит в основе метода НВИ [5]. Такое последовательное сравнение результатов воздействия зондирующих импульсов оказалось эффективным способом контроля состояния обмоток трансформаторов. Метод НВИ практически с момента изобретения приобрел широкую популярность и интенсивно развивался во всех высоковольтных лабораториях и электроэнергетических системах. Существенное развитие метода НВИ состоялось в 70-80 гг. ХХ в. во Всесоюзном Электротехническом Институте им. Ленина [6-8]. Метод НВИ в результате детальных исследований был значительно усовершенствован и получил название «импульсное дефектографирование» [6-9]. Технология импульсного дефектографирования успешно внедрялась на трансформаторных заводах и энергосистемах РФ $[10,11]$. Однако сложность воспроизведения измерений, погрешности при оценке состояния обмоток на основе анализа сигналов отклика и необходимость в наличии нормограмм - откликов обмоток в исправном состоянии - привели к необходимости модернизации метода НВИ. В связи с этим в конце XX в. в лабораториях Северной Америки метод НВИ был трансформирован в технологию, получившую название «метод частотных характеристик», или МЧА. Отличие от первоначального или «классического» метода НВИ состоит в принципиальной другой форме и длительности как зондирующего импульса, так и сигнала отклика. Если в методе НВИ используется зондирующий импульс длительностью от сотен миллисекунд до единиц микросекунд, то в методе МЧА применяется сигнал амплитудой около 10 В, частота которого плавно изменяется от единиц Гц до нескольких МГц. В свою очередь, отклик, представляющий собой короткий импульс в методе НВИ, в технологии МЧА есть амплитудно-частотная характеристика обмотки, представляющая собой реакцию на сигнал, подаваемый на соседнюю обмотку. МЧА позволил преодолеть ряд недостатков, свойственных методу НВИ, а именно, снизилась зависимость от точности схемы воспроизведения измерений. Вместе с этим возросла корректность результатов контроля состояния обмоток, так как сравнение частотных характеристик, выполняемое с помощью программных средств, обладает меньшей погрешностью, чем сравнение осциллограмм импульсных откликов, выполняемое визуально. Поэтому на сегодняшний день технология МЧА является основной в электроэнергетических системах США, Канады, Бразилии, Евросоюза, Японии, Южной Кореи, Китая и многих других стран [12-17].

Не смотря на все преимущества и высокий производственный потенциал МЧА, ошибки при выявле- 
нии дефектов и постановки диагноза встречаются достаточно часто при использовании и метода НВИ, и МЧА [1-4]. Поэтому вопрос о разработке достоверной технологии качественной диагностики обмоток остается актуальным.

В лаборатории диагностики и испытаний Инженерной школы энергетики ТПУ исследуется способ увеличения эффективности традиционного метода низковольтных импульсов. Одним из путей этого направления исследований является использование зондирующего импульса по форме, близкой к прямоугольной и имеющей длительность десятки-сотни наносекунд. Результаты экспериментальных исследований, демонстрирующие перспективность пути трансформирования импульсного метода в направлении уменьшения длительности зондирующего импульса, приведены в [18-21].

В энергосистемах Евросоюза, Америки и ЮгоВосточной Азии МЧА считается наиболее предпочтительным и достоверным [14-17]. Однако отсутствие возможности диагностики под рабочим напряжением и необходимость наличия нормограмм не позволяют считать МЧА технологией завтрашнего дня.

Среди основных тенденций развития процесса контроля состояния обмоток выделяются два основных - выявление дефектов на ранней стадии развития и контроль состояния обмоток без снятия рабочего напряжения и расшиновки трансформатора (режим «ON-LINE»). На исследование физических основ и технической реализации технологии, отвечающей указанным требованиям, направлены представленные исследования.

\section{Постановка задачи исследования}

Задача исследования - экспериментально изучить принципиальную возможность контролировать состояние обмотки и обнаруживать дефект, используя включение трансформатора в сеть, при этом коммутационный импульс, поступающий на обмотку в результате штатной коммутации, является зондирующим импульсом. Эксперименты проводились на реальном трехфазном трансформаторе, у которого две фазы исправны и не содержат никаких дефектов, а на одной фазе создано дефектное состояние. По откликам на воздействие коммутационного импульса необходимо выяснить, определяется ли наличие дефекта по отклику, и исследовать эффективность указанного подхода при подаче коммутационного импульса на различные обмотки трансформатора, наиболее оптимальное место регистрации сигнала отклика, при наличии и в отсутствии нагрузки различного характера.

На обмотках низкого напряжения $(\mathrm{HH})$ и высокого напряжения (BН) фазы А трансформатора НТМИ-6-66 было создано дефектное состояние, как показано на рис. 1.

Для подачи коммутационного импульса сети на исследуемую обмотку в определенный момент времени по фазе напряжения реализована схема подключения трансформатора и регистрация отклика при коммутации. Схема приведена на рис. 2.

Элементы схемы функционируют следующим образом. При поступлении напряжения от сети 220 B,
50 Гц на ЛАТР возникает напряжение на вторичной обмотке трансформатора OCM, VD выпрямляет ток от трансформатора, конденсатор $C$ заряжается. По мере заряда напряжение на конденсаторе увеличивается. При достижении значения напряжения, соответствующего открытию динистора, динистор открывается, и ток, проходящий через динистор $V S_{1}$, открывает тиристор $V S_{2}$. Коммутационный импульс напряжения, используемый как зондирующий, подаётся на испытуемый объект - обмотку трансформатора. Изменение задержки подачи импульса осуществляется с помощью изменения сопротивления резисторов и ёмкости $C$. Регистрация зондирующего импульса и отклика обмоток производится при помощи цифрового двухканального осциллографа Tektronix TDS 2012.

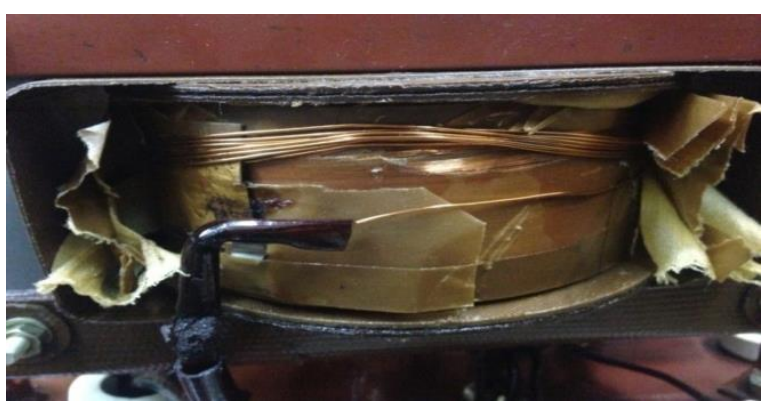

Pис. 1. Дефект обмотки ВН - аксиальное смещеене обмотки

Fig. 1. Fail of high voltage winding $(H V)$ - winding axial shift

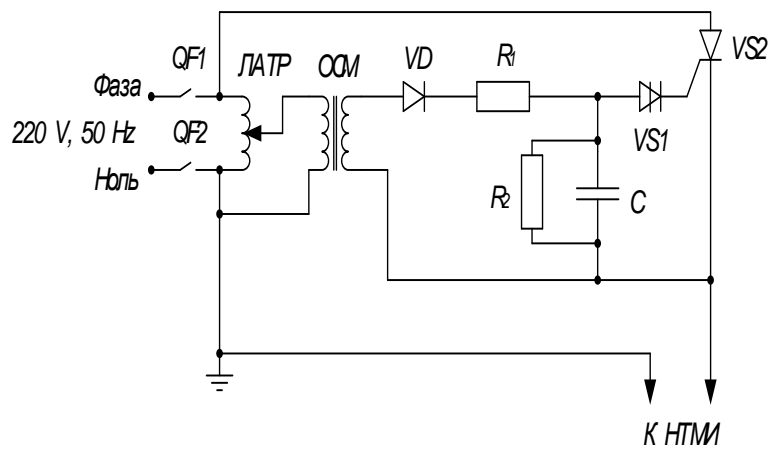

Pис. 2. Принципиальная схема блока коммутации: $Q F$ автоматические выключатели; ЛАТР - лабораторный автотрансформатор; ОСМ - понижающий трансформатор питания блока управления тиристром ОСM1-0,1; VD - выпрямительный диод $1 N 4007 ; R_{1}, R_{2}$ - резисторы, позволяющие ограничивать ток заряда конденсатора; $C$ конденсатор; $V S_{1}-$ динистор $K H 102 \mathrm{~A} ; \mathrm{VS}_{2}-$ тиристор КУ202Н; НТМИ - исследуемый объект

Fig. 2. Principal scheme of commutation block: $Q F-a u$ tomatic switches; ЛАTP - laboratory autotransformer; OCM - set down transformer of thyristor control block OCM1-0,1; VD - diode 1N4007; $R_{1}$, $R_{2}-$ resistors for capacitor current restriction; $C$ condenser; $V S_{1}$ - dynistor $K H 102 A ; V S_{2}-$ thyristor КУ202H; НТМИ-researched object

\section{Экспериментальная часть}

1. Коммутационная операция «включение» от сети 220 В на стороне НН. Отклик регистрируется на стороне ВН одноименной фазы с помощью ем- 
костного делителя напряжения. Контроль обмоток по схеме «НН-ВН». Осциллограммы результатов диагностики приведены ниже. Отклики неповрежденных пар в-В и с-С существенно отличаются от ситуации для пары а-А. Наблюдение сигналов

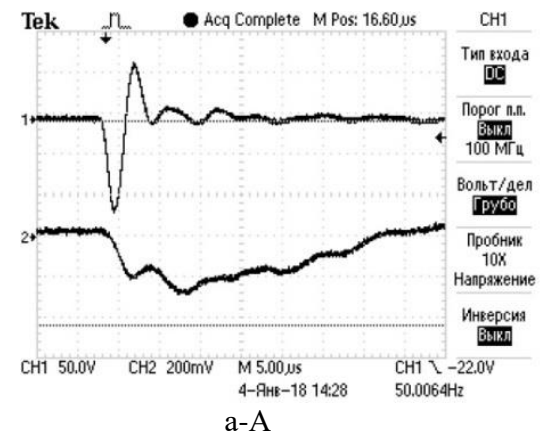

отклика проводилось на развертке 5 мкс на деление для наблюдения начальной стадии переходного процесса (рис. 3), на развертке 25 мкс на деление для наблюдения всего переходного процесса в обмотке (рис 4).

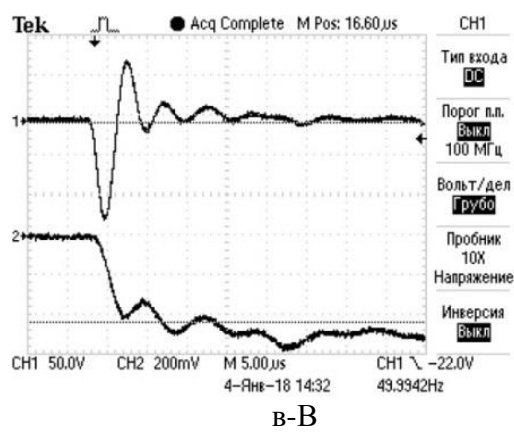

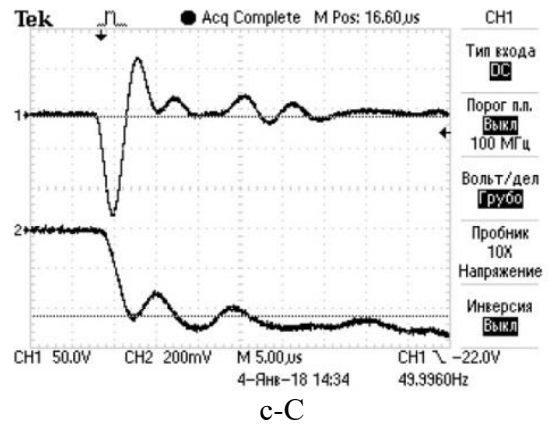

Pис. 3. Осциллограммы коммутационного импульса (луч 1) и сигналов отклика (луч 2) для различныхх фаз. Развертка осииллографа - 5 мкс/деление

Fig. 3. Waveforms of commutation pulse (beam 1) and response (beam 2) for different phases. Oscilloscope sweep - 5 microsecond per scale division

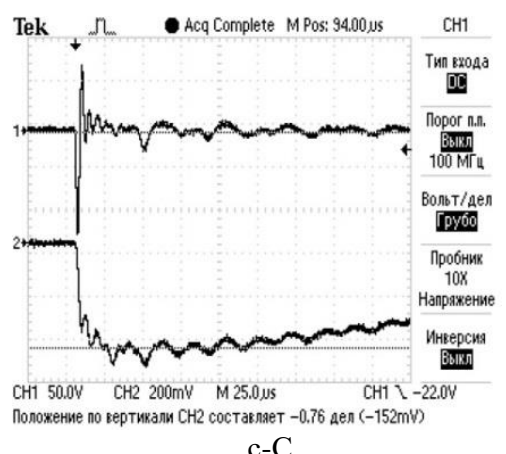

$\mathrm{c}-\mathrm{C}$

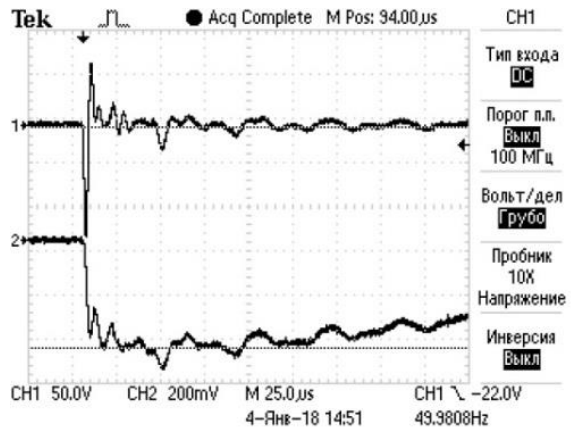

B-B

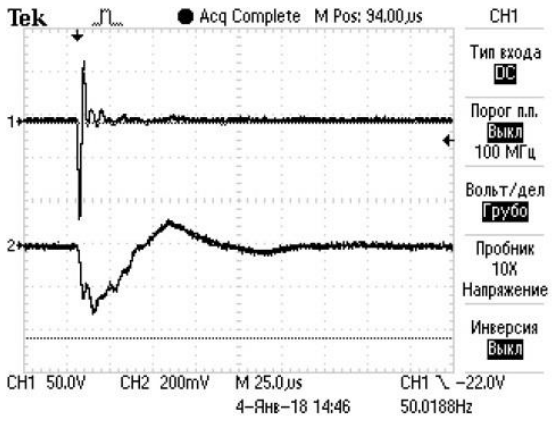

a-A

Рис. 4. Осциллограммы коммутачионного импульса (луч 1) и сигналов отклика (луч 2) для различных фаз. Развертка осииллографа - 25 мкс/деление

Fig. 4. Waveforms of commutation pulse (beam 1) and response (beam 2) for different phases. Oscilloscope sweep - 25 microsecond per scale division 
Визуальный анализ полученных осциллограмм без применения специальных программ сравнения позволяет констатировать факт отличия откликов на поврежденной фазе при идентичности откликов двух неповрежденных фаз и равенстве импульса коммутации при включении.

2. Контроль состояния обмотки с подключенной нагрузкой на стороне НН. Нагрузку моделирует реостат. Максимальное сопротивление реостата

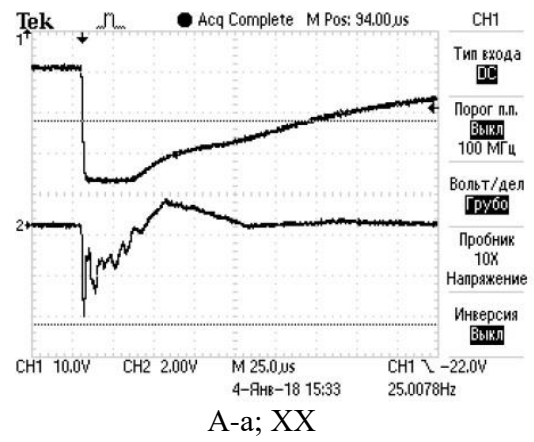

40 Ом. Наблюдение осциллограммы отклика по схеме «ВН-НН» (Коммутационная операция «включение» от сети 220 В на стороне ВН). Наблюдаемые осциллограммы приведены на рис. 5 для различных значений резистивных нагрузок вторичной обмотки трансформатора. На рис. 6 приведены аналогичные осциллограммы откликов при различных значениях емкостных нагрузок вторичной обмотки трансформатора.

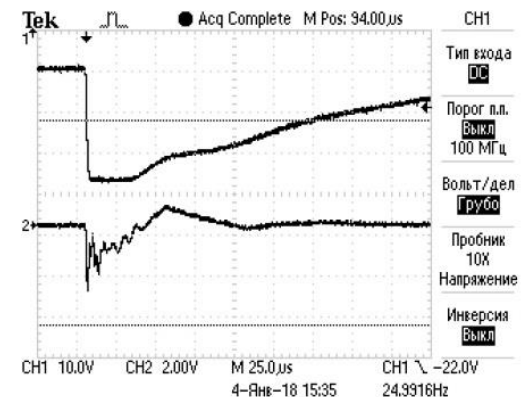

А-а; нагрузка $100 \%$

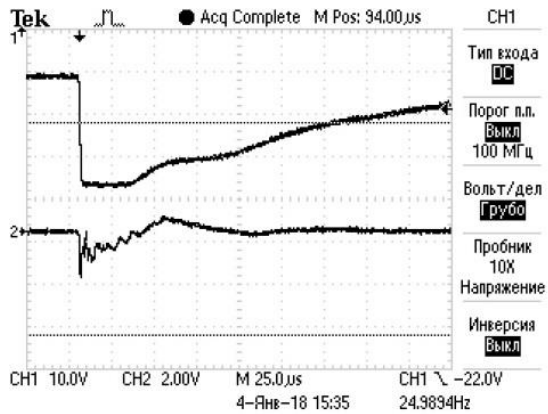

A-а; нагрузка $50 \%$

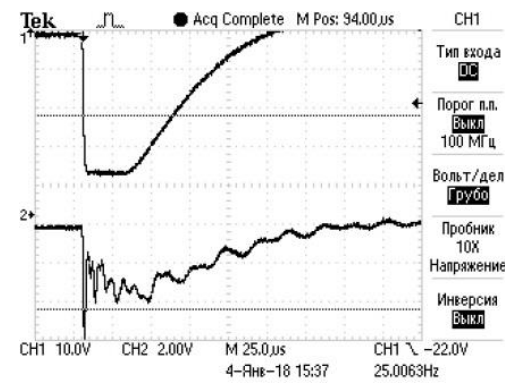

В-в; нагрузка $100 \%$

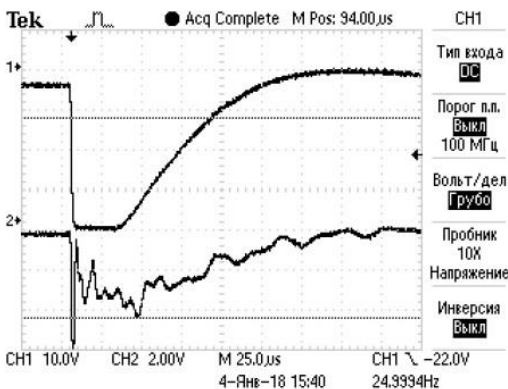

C-c; нагрузка $100 \%$

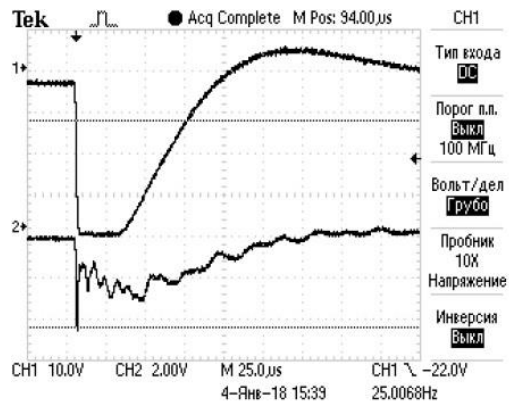

В-в; нагрузка 50 \%

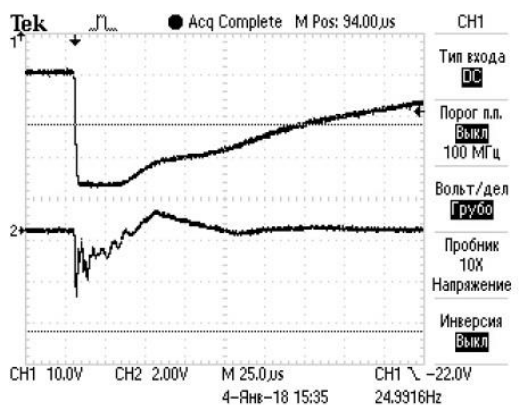

A-а; нагрузка $50 \%$

Рис. 5. Осциллограммы коммутационного импульса (луч 1) и сигналов отклика (луч 2) для различных фаз и различныхх значений резистивной нагрузки низковольтной обмотки трансформатора. Развертка осциллографа - 25 мкс/деление

Fig. 5. Waveforms of commutation pulse (beam 1) and response (beam 2) for different phases and different resistive loads of low voltage winding. Oscilloscope sweep - 25 microsecond per scale division 


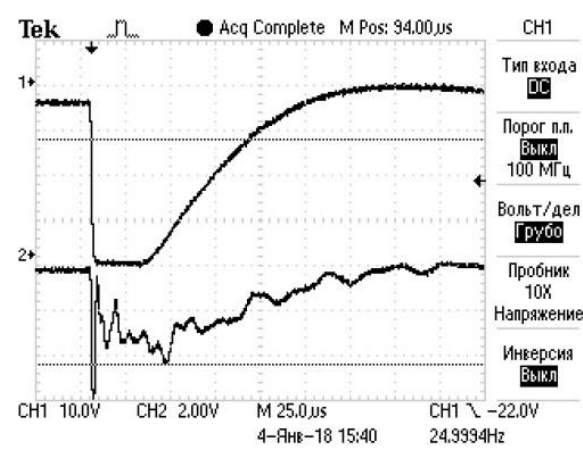

C-a; нагрузка - емкость 0,05 мкФ

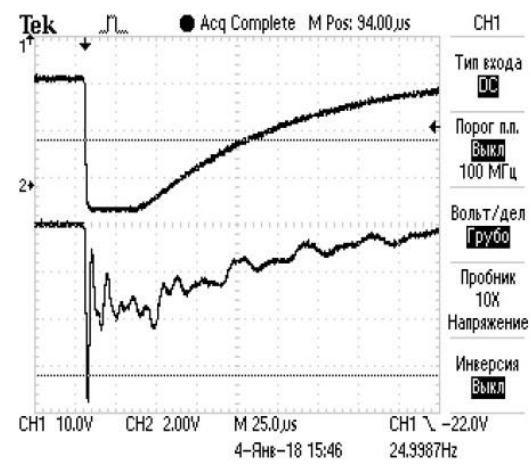

$\mathrm{C}$-а; нагрузка - емкость 0,15 мкФ

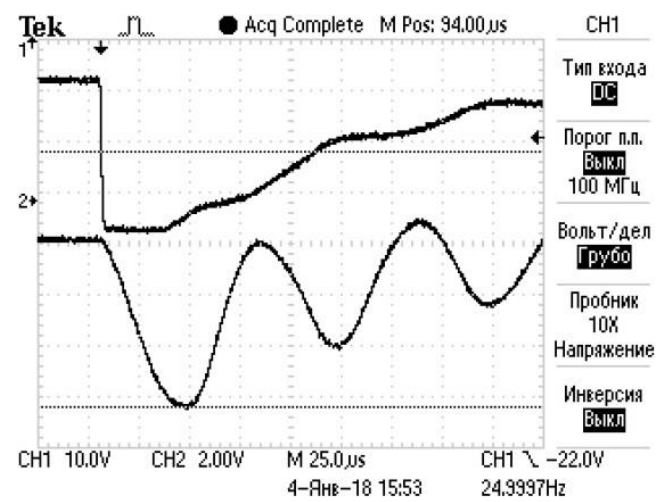

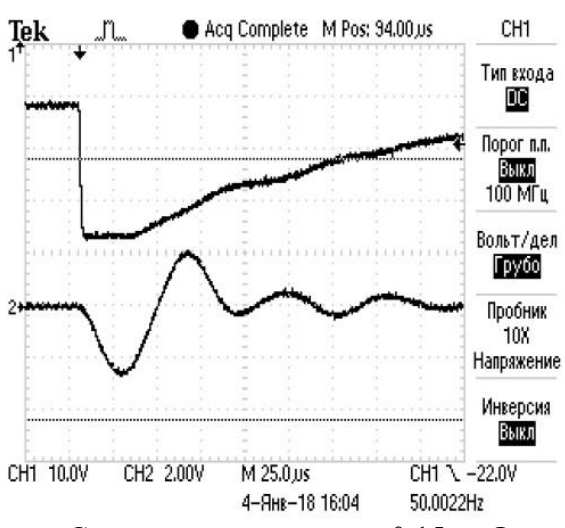

С-с; нагрузка - емкость 0,15 мкФ

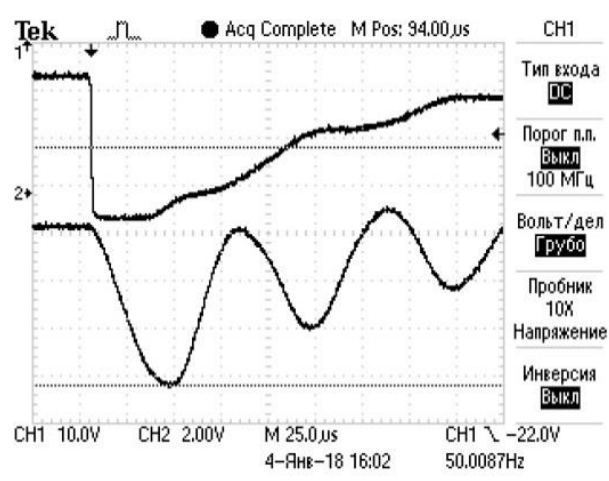

С-с; нагрузка - емкость 0,05 мкФ

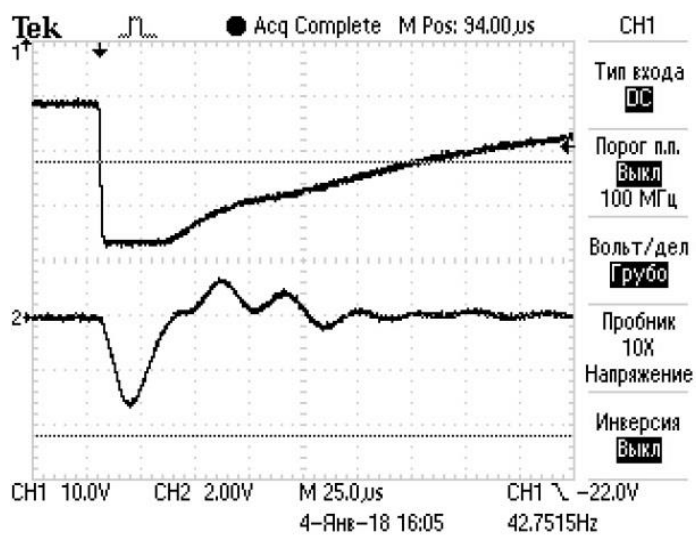

A-а; нагрузка - емкость 0,15 мкФ

Pис. 6. Осциллограммы коммутационного импульса (луч 1) и сигналов отклика (луч 2) для различных фаз и различных значений емкостной нагрузки вторичной обмотки трансформатора. Развертка осциллографа 25 мкс/деление

Fig. 6. Waveforms of commutation pulse (beam 1) and response (beam 2) for different phases and different capacitive loads of low voltage winding. Oscilloscope sweep - 25 microsecond per scale division 


\section{Обсуждение результатов}

Основной задачей экспериментов, описанных выше, являлась демонстрация принципиальной возможности контролировать развитие дефектного состояния обмотки. В качестве критерия определения дефектного состояния принимается различие в форме сигналов отклика при сравнении откликов с фаз А и $\mathrm{B}, \mathrm{A}$ и $\mathrm{C}$ при идентичности откликов для фаз В и С, при идентичном коммутационном импульсе, выполняющем роль зондирующего. На всех представленных осциллограммах зондирующий импульс - луч 1. Аксиальное смещение витков было организовано на фазе А.

Отклики неповрежденных пар, соответствующие лучу $2,-$ в-B и с-С - существенно отличаются от ситуации для пары а-А для случаев отсутствия или наличия нагрузки. Причем как для емкостной, так и для резистивной нагрузок ситуация повторяется. Наблюдения сигналов отклика проводилось на развертке 5 мкс на деление для наблюдения начальной стадии переходного процесса (рис. 3), на развертке 25 мкс на деление для наблюдения всего переходного процесса в обмотке (рис. 4-6).

Для определения потенциальной эффективности предложенного метода контроля состояния обмоток использовался визуальный контроль осциллограмм. Для более детальной оценки полученных осциллограмм могут быть применены современные программные средства, предполагающие разложение в ряд Фурье и спектральный анализ. Однако именно факт четкого различия в откликах здоровых и повре-

\section{СПИСОК ЛИТЕРАТУРЫ}

1. Соколов В.В. Ранжирование состаренного парка силовых трансформаторов по техническому состоянию // Современное состояние и проблемы диагностики силового электрооборудования: Материалы IV Всероссийской научно-технической конференции. - Новосибирск, 26-28 сентября, 2006. - С. 7-18

2. Pettersson L., Fantana N.L., Sunderman U. Assessment ranking of power transformers using condition based evaluation, a new approach // CIGRE Paris Conference. - Paris, France, 1998. Paper 12-204.

3. Осотов В.Н. О методологии оценки состояния электрообору дования с большим сроком службы // Современное состояние и проблемы диагностики силового электрооборудования: Материалы IV Всероссийской научно-технической конференции. - Новосибирск, 26-28 сентября, 2006. - С. 25-29.

4. Вдовико В.П. Диагностика высоковольтного электрооборудования и эффективность ее применения // Современное состояние и проблемы диагностики силового электрооборудования: Материалы IV Всероссийской научно-технической конференции. - Новосибирск, 26-28 сентября, 2006. - С. 34-41.

5. Лех В., Тымински Л. Новый метод индикации повреждений при испытании трансформаторов на динамическую прочность // Электричество. - 1966. - Т. 1. - № 1. - С. 77-81.

6. Аветиков Г.В., Левицкая Е.И, Попов Е.А. Импульсное дефектографирование трансформаторов на при испытаниях на электродинамическую стойкость // Электротехника. - 1978. - № 4. - С. 53-57.

7. Конов Ю.С., Короленко В.В., Федорова В.П. Обнаружение повреждений трансформаторов при коротких замыканиях // Электрические станции. - 1980. - № 7. - С. 46-48.

8. Количественная оценка результатов импульсного дефектографирования обмоток силовых трансформаторов / С.В. Аликин, А.А. Дробышевский, Е.И. Левицкая, М.А. Филатова // Электротехника - 1990. - № 5. - С. 75-76.

9. Диагностика обмоток силовых трансформаторов методом низковольтных импульсов / С.В. Аликин, А.А. Дробышевский, Е.И. Левицкая, М.А. Филатова // Электротехника. - 1991. № 12 . - C. $30-35$. жденной обмоток без использования дополнительных математических средств, программных инструментов и специального зондирующего генератора является подтверждением эффективности предложенного метода контроля состояния.

\section{Заключение}

Проблема контроля состояния активных частей трансформаторов существует с момента создания высоковольтных электроэнергетических систем. Развитие дефектов обмоток происходит неконтролируемо и в результате заканчивается выходом трансформатора из строя. К настоящему моменту создан широкий спектр методов и средств контроля состояния обмоток, однако все они требуют снятия напряжения и расшиновки трансформатора. Создание технологии контроля состояния обмоток под рабочим напряжением позволит вывести диагностику трансформаторов на новый технологический уровень. Одним из путей решения проблемы контроля состояния без снятия напряжения является использование коммутационных импульсов сети в качестве зондирующих. Показано, что достоверный контроль состояния обмотки возможен при дефекте аксиальное смещение витков обмотки.

Технология контроля состояния обмоток на основе коммутационных импульсов, в качестве зондирующих, является информативной и потенциально превосходит все существующие методы и средства диагностики обмоток.

10. Хренников А.Ю., Киков О.М. Диагностика силовых трансформаторов в Самараэнерго методом низковольтных импульсов // Электрические станции. - 2003. - № 11. - С. 49-51.

11. Хренников А.Ю. Опыт обнаружения остаточных деформаций обмоток силовых трансформаторов // Энергетик. - 2003. № 7. - C. 18-20.

12. Diagnosis of interturn faults of single-distribution transformers under controlled conditions during energization / D. Guillen, J. Olivares-Galvan, R. Escarela-Perez, D. Granados-Lieberman, E. Barocio // Measurement. - 2019. - V. 141. - P. 24-36.

13. High frequency electric circuit modeling for transformer frequency response analysis studies / X. Zhaoa, C. Yaoa, A. Abu-Siadab, R. Liaoa // Electrical Power and Energy Systems. - 2019. V. 111. - P. 351-368.

14. Diagnostic of transformer winding deformation fault types using continuous wavelet transform of pulse response / Q. Cheng, Z. Zhao, C. Tang, G. Qian, S. Islam // Measurement. - 2019. V. 140. - P. 197-206.

15. Velasquez R., Lara J., Melgar A. Converting data into knowledge for preventing failures in power transformers // Engineering Failure Analysis. - 2019. - V. 101. - P. 215-229.

16. Zarkovi M., Stojkovi Z. Analysis of artificial intelligence expert systems for powertransformer condition monitoring and diagnostics // Electric Power Systems Research. - 2017. V. 149. - P. 125-136.

17. Senobari R., Sadeh J., Borsi H. Frequency response analysis (FRA) of transformers as a tool for fault detection and location: a review // Electric Power Systems Research. - 2019. - V. 155. - P. 172-183.

18. Lavrinovich V.A., Lavrinovich A.V., Mytnikov A.V. Development of advanced control state technology of transformer and electric motor windings based on pulsed method // International Journal on Technical and Physical Problems of Engineering. - 2012. - V. 4. - № 4. - P. 149-153.

19. Lavrinovich V.A, Isaev Y.N., Mytnikov A.V. Modeling of state control procedure of power transformer winding by short probe pulses // International Journal on Technical and Physical Problems of Engineering. - 2014. - V. 6. - № 1. - P. 145-147. 
20. Lavrinovich V.A, Mytnikov A.V. Development of pulsed method for diagnostics of transformer windings based on short probe impulse // IEEE Translation on Dielectric Electrical Insulation. 2015. - V. 22. - № 4. - P. 2041-2045.

21. Lavrinovich V.A., Mytnikov A.V., Hongda Li. Advanced technology of transformer winding condition control based on

\section{Информация об авторах}

Лавринович В.А., доктор технических наук, профессор, главный конструктор по электротехническому оборудованию, Российский Федеральный Ядерный Центр - Всероссийский Научно-Исследовательский Институт Технической Физики - Всероссийский Электротехнический Институт.

Лавринович A.B., инженер 1 категории, отдел по высоковольтному оборудованию, Томский научноисследовательский и проектный институт нефти и газ.

Мытников A.B., кандидат технических наук, доцент отделения электроэнергетики и электротехники Инженерной школы энергетики Национального исследовательского Томского политехнического университета. 
UDC 621.314.21-027.3.04

\title{
EXPERIMENTAL RESEARCH OF CONTROLLING HIGH VOLTAGE TRANSFORMER WINDING CONDITION BASED ON SWITCHING IMPULSES
}

\author{
Valeriy A. Lavrinovich ${ }^{1}$, \\ lavrhome@mail.ru
}

\author{
Alexey V. Lavrinovich², \\ lavrinovich86@yandex.ru
}

\author{
Alexey V. Mytnikov³, \\ mytnikov66@mail.ru \\ 1 Russian Federal Nuclear Center - All-Russia Research Institute of technical Physics, \\ 12, Krasnokazarmennaya street, Moscow, 111250, Russia. \\ 2 Tomsk research and design institute of oil and gas, \\ 72, Mira avenue, Tomsk, 634027, Russia. \\ 3 National Research Tomsk Polytechnic University, \\ 30, Lenin Avenue, Tomsk, 634050, Russia.
}

\begin{abstract}
The importance of the research is driven by the exceptional importance of stable work of electrical equipment complex which is used at production, transportation and processing of geo-resources. Effectiveness and reliability of such geological production strongly depend on high voltage transformer condition. Failure of such a transformer can cause serious breakage of the whole electrical energy system and even breakdown. This, in its turn, leads to serious ecological and material losses. Fail of active transformer part, especially winding, is one of the reasons of accident due to the influence of over-voltage waves and short circuit electro-dynamic forces on the winding. To prevent emergence situations with high voltage transformers and production stop losses an effective technology of winding condition control is necessary. Standard methods are ineffective for detecting winding fails at early stages of development. Among the new ones and those recommended by the international expert institutes of IEC and CIGRE are modifications of the pulse method, primarily the frequency analysis method. But, despite the recognition as the most reliable and promising, this method does not always allow revealing fails of the windings, especially in the early stages of development. Moreover, a prerequisite for the use of frequency analysis method technology requires the complete removal of voltage, the transformer spacing and the use of special monitoring equipment. At the same time, to obtain reliable diagnostic results, it is necessary to have special reference signals - normograms, which are absent practically for the whole range of power transformers. These factors reduce the common effectiveness of frequency analysis method technology. That is why, at present, the issue of research and development of technology for monitoring the state of windings under operating voltage is fully relevant.

The main aim of the study is an experimental research of the principal possibility of monitoring the transformer winding condition on the basis of switching processes in the high voltage grid.

Objects of the research are the windings of real transformer in two conditions - with and without fault.

Methods: the simulation method on a real physical object. In the transformer NTMI-6 a fail of the mechanical winding shift was developed on one phase. The other two phases are in normal condition. With the help of a special circuit, the transformer was connected to the network. Using a Tektronix TDS-2012 electronic oscilloscope, the voltage on the primary winding and the response signal from the samename secondary winding were measured. The measurements were repeated for the other two phases in the same way.

Results. On a real transformer of the NTMI-6 type, the principal possibility of controlling high voltage transformer winding condition by means of switching impulses was experimentally researched. It is established that monitoring of mechanical state of windings using the switching impulses could be effectively realized. It is shown that when the transformer is turned on in the network, the operating voltage can serve as a diagnostic impulse suitable for probing the mechanical condition of the transformer windings using the low-voltage impulse method.
\end{abstract}

\section{Key words:}

Condition control, pulsed method, probing impulse, winding fail, diagnostics efficiency.

\section{REFERENCES}

1. Sokolov V.V. Ranzhirovanie sostarennogo parka silovykh transformatorov po tekhnocheskomu sostoyaniyu [Ranking of the aged power transformer park due to technical technical condition]. Sovremennoe sostoyanie i problem diagnostiki silovogo elektrooborudovaniya. Materialy IV Vserossiyskoy nauchno-tekhnicheskoy konferentsii [Modern condition and problems of power electrical equipment diagnostics. Proc. of IV All-Russian scientific conference]. Novosibirsk, Russia, 2006. pp. 7-18.

2. Pettersson L., Fantana N.L., Sunderman U. Assessment ranking of power transformers using condition based evaluation, a new approach. CIGRE Paris Conference. Paris, France, 1998. Paper 12-204,

3. Osotov V.N. O metodologii otsenki sostoyaniya elektrooborudovaniya $\mathrm{s}$ bolshim srokom sluzhby [On methodology of electrical equipment estimation with large term in service]. Sovremennoe sostoyanie i problem diagnostiki silovogo elektrooborudovaniya. Materialy IV Vserossiyskoy nauchno-tekhnicheskoy konferentsii [Modern condition and problems of power electrical equipment diagnostics. Proc. of IV All-Russian scientific conference]. Novosibirsk, Russia, 2006. pp. 25-29.

4. Vdoviko V.P. Diagnostika vysokovoltnogo elektrooborudovaniya i effektivnost ee primeneniya [Diagnostics of high voltage equipment and effectiveness of its application]. Sovremennoe sostoyanie $i$ problemy diagnostiki silovogo elektrooborudovaniya. Materialy IV Vserossiyskoy nauchno-tekhnicheskoy konferentsii [Modern condition and problems of power electrical equipment diagnostics. Proc. of IV All-Russian scientific conference]. Novosibirsk, Russia, 2006. pp. 34-41. 
5. Lekh V., Tyminskiy L. New method of fail diagnostics at the dynamic reliability transformer test. Electricity, 1966, vol. 1, no. 1, pp. 77-81. In Rus.

6. Avetikov G.V., Levitskaya E.I., Popov E.A. Impulsed defectography of transformers at the electrodynamic reliability test. Electrotechnic, 1978, no. 4, pp. 53-57. In Rus.

7. Konov Yu.S., Korolenko V.V., Fedorova V.P. Obnaruzhenie povrezhdenii transformatorov pri korotkikh zamykaniyakh [Fail detection of transformers due to short circuit]. Elektricheskie Stantsii, 1980, no. 7, pp. 46-48.

8. Alikin S.V., Drobyshevskiy A.A., Levitschaya E.I., Filatova M.A Kolichesvennaya oshcenka rezultatov impulsnogo defektografirovaniya obmotok silovykh transformatorov [Quantitative assessment of the of pulse defectography results of power transformer windings]. Elektrotechnika, 1990, no.5, pp. 75-76. In Rus.

9. Alikin S.V., Drobyscevskiy A.A., Levitskaya E.I., Filatova M.A. Power transformer winding diagnostics by low-voltage pulsed method. Electrotechnic, 1991, no. 12, pp. 30-35. In Rus.

10. Khrennikov A.Yu., Kikov O.M. Diagnostika silovykh transformatorov v Samaroenergo metodom nizkovoltnykh impulsov [Power transformer diagnostics by low-voltage pulsed method in Samaropenergo]. Elektricheskie Stantsii, 2003, no. 11, pp. 49-51.

11. Khrennikov A.Yu. Opyt obnaruzheniya ostatochnykh deformatsy obmotok silovykh trasnformatorov [Experience of mechanical deformation detection of power transformer windings]. Energetic, 2003, no. 7, pp. 18-20.

12. Guillen D., Olivares-Galvan J., Escarela-Perez R., GranadosLieberman D., Barocio E. Diagnosis of interturn faults of singledistribution transformers under controlled conditions during energization. Measurement, 2019, vol. 141, pp. 24-36.

13. Zhaoa X., Yaoa C., Abu-Siadab A., Liaoa R. High frequency electric circuit modeling for transformer frequency response analysis studies. Electrical Power and Energy Systems, 2019, vol. 111, pp. 351-368.
14. Cheng Q., Zhao Z., Tang C., Qian G., Islam S. Diagnostic of transformer winding deformation fault types using continuous wavelet transform of pulse response. Measurement, 2019, vol. 140, pp. 197-206.

15. Velasquez R., Lara J., Melgar A. Converting data into knowledge for preventing failures in power transformers. Engineering Failure Analysis, 2019, vol. 101, pp. 215-229.

16. Zarkovi M., Stojkovi Z. Analysis of artificial intelligence expert systems for powertransformer condition monitoring and diagnostics. Electric Power Systems Research, 2017, vol. 149, pp. $125-136$.

17. Senobari R., Sadeh J., Borsi H. Frequency response analysis (FRA) of transformers as a tool for fault detection and location: a review. Electric Power Systems Research, 2019, vol. 155, pp. 172-183.

18. Lavrinovich V.A., Lavrinovich A.V., Mytnikov A.V. Development of advanced control state technology of transformer and electric motor windings based on pulsed method. International Journal on Technical and Physical Problems of Engineering, 2012, vol. 4, no. 4, pp. 149-153.

19. Lavrinovich V.A., Isaev Y.N., Mytnikov A.V. Modeling of state control procedure of power transformer winding by short probe pulses. International Journal on Technical and Physical Problems of Engineering, 2014, vol. 6, no. 1, pp. 145-147.

20. Lavrinovich V.A., Mytnikov A.V. Development of pulsed method for diagnostics of transformer windings based on short probe impulse. IEEE Translation on Dielectric Electrical Insulation, 2015, vol. 22, no. 4, pp. 2041-2045.

21. Lavrinovich V.A., Mytnikov A.V., Hongda Li. Advanced technology of transformer winding condition control based on nanosecond probing impulse. Resource-Efficient Technologies, 2016, vol. 2, no. 3, pp. 111-117.

Received: 14 November 2019.

\section{Information about the authors}

Valeriy A. Lavrinovich, Dr. Sc., professor, Russian Federal Nuclear Center - All-Russia Research Institute of technical Physics.

Alexey V. Lavrinovich, engineer of first category, Tomsk research and design institute of oil and gas.

Alexey V. Mytnikov, Cand. Sc., associate professor, National Research Tomsk Polytechnic University. 